\title{
Rings with Central Idempotent or Nilpotent Elements
}

\author{
By M. P. Drazin \\ (Received 11th June, 1956.)
}

\section{Introduction.}

It is easy to see ( $c f$. Theorem 1 below) that the centrality of all the nilpotent elements of a given associative ring implies the centrality of every idempotent element; and (Theorem 7) these two properties are in fact equivalent in any regular ring. We establish in this note various conditions, some necessary and some sufficient, for the centrality of nilpotent or idempotent elements in the wider class of $\pi$-regular rings (in Theorems 1, 2, 3 and 4 the rings in question are not even required to be $\pi$-regular).

We discuss particularly the special case of a ring $R$ (possibly with operators) having minimal condition on (say) left ideals; such an $R$ is necessarily $\pi$-regular (see [1]). It is well known that, if a given left ideal $A$ of $R$ contains no non-zero idempotent, then $A$ must be nilpotent; and of course the converse of this is obvious (in any ring whatever). We consider in our concluding section what can be said along these lines if we replace the nilpotency of $A$ by the weaker condition that $R$ contains a non-zero $A$-annihilator. For any non-zero left ideal $A$ whose idempotent elements are all central (in $A$ ) we obtain the following analogue: if $A$ contains no element acting as a two-sided identity on $A$, then $A$ contains a two-sided $A$-annihilator (the converse again being trivial). This leads at once to some simple sufficient conditions for the existence of one-sided ${ }^{*}$ $A$-annihilators.

Our arguments throughout are of a very straightforward and elementary nature, but the results do nevertheless seem worth putting on record. For brevity, we shall call a given associative ring (or algebra) $R$ a $C N$-ring whenever every nilpotent element of $R$ is central, and a $C I$-ring whenever every idempotent element of $R$ is central; when we speak of $A$ as being a $C I$-ideal of $R$, we shall mean that $A$ is an ideal of $R$ and that, considered as a ring in its own right, $A$ is itself a $C I$-ring. Finally, given any two elements $u, v$ of a ring, we shall denote their additive commutator $u v-v u$ by $[u, v]$. 


\section{General results.}

In this section we shall be concerned with results for rings not subjected to any "finiteness restrictions". We first formally establish the result mentioned in the opening sentence of the Introduction; special cases of this have previously been noted by Forsythe and McCoy ([7], Lemma 1) and Herstein ([8], Lemma 4).

\section{THEOREM 1. Every $C N$-ring is a $C I$-ring.}

Proof. Let $v$ be any given element of a given associative ring $R$, let $e$ be any idempotent element of $R$, and define $z=e v-e v e$. Then $z e=0$, and so $z^{2}=z e(v-v e)=0$; thus, if $R$ is a $C N$-ring, then $z$ is central, and in particular $0=[e, z]=e z=z$, i.e. we have $e v=e v e$. Also, by a precisely similar argument, $v e=e v e$, and so $e v=v e$. Since $v$ was arbitrary, consequently $e$ is central; but $e$ was an arbitrary idempotent in $R$, and so the result follows.

Certain other classes of $C I$-rings are described in Section 3 of [5].

Our next theorem is due to A. Rosenberg (private communication), and concerns rings of a type of which several special cases have been considered recently (see e.g. [5], [6], [8]).

THeOREM 2. If, to each pair of elements $x, y$ of a given ring $R$, there corresponds an element $a(x, y)$ of $R$ such that $\left[x-x^{2} a(x, y), y\right]=0$, then $R$ is a $C N$-ring.

Proof. Let $A_{t}(x)$ denote the two-sided ideal of $R$ generated by $x^{t}(t=1,2, \ldots)$. It clearly suffices to show that, given any $x, y \in R$, there are elements $h_{t}(x, y) \varepsilon A_{t}(x)$ satisfying $\left[x-h_{t}(x, y), y\right]=0 \quad(t=2,3, \ldots)$; we may of course take $h_{2}(x, y)=x^{2} a(x, y)$, and we shall obtain the result by induction, showing that, if $h_{t}(x, y)$ exists for a given $t$, then, on writing $\dot{h}_{t}(x, y)=h$, we may take $h_{t+1}(x, y)=h^{2} a(h, y)$.

Of course $[x-h, y]=0$, while, by our hypothesis on $R$,

$$
\left[h-h^{2} a(h, y), y\right]=0 \text {; }
$$

thus, by addition, $\left[x-h^{2} a(h, y), y\right]=0$, so, to set up our induction, we need only show that $h^{2} a(h, y) \varepsilon A_{t+1}(x)$. And we in fact even have $\dot{h}^{2} a(h, y) \varepsilon A_{2 t}(x)$. For, since $h \varepsilon A_{t}(x)$, we know that $h^{2} a(h, y)$ is a sum of terms of the forms $u x^{t} v x^{t} w, x^{t} v x^{t} w, u x^{2 t} w$ with $u, v, w \varepsilon R$; any term of the last type is obviously in $A_{2 t}(x)$, so it now only remains to consider terms of the form $q=x^{t} v x^{t}$, and, since $\left[x^{t}-\left(x^{t}\right)^{2} a\left(x^{t}, v\right), v\right]=0$, we have $q=\left(v x^{t}+\left[x^{2 t} a\left(x^{t}, v\right), v\right]\right) x^{t} \varepsilon A_{2 t}(x)$. 
Lest Theorem 2 should appear a trifle special or artificial, we mention here that the condition which it asserts to be sufficient for $R$ to be a $C N$-ring is, for a wide class of rings (including all finite-dimensional algebras), in fact also necessary; this will appear in Theorem 5 below. Our last result in this section, which dates back as far as Peirce ( $c f$. also [7], Lemma 2 and [8], Lemma 9), is only mildly relevant to the subject in hand, but we include it now for completeness; we shall call an idempotent element $e$ of a ring $R$ trivial (in $R$ ) if either $e=0$ or $e$ acts as a two-sided identity on $R$.

THEOREM 3. In any directly irreducible ring, every central idempotent is trivial.

Proof. Let $e$ be any non-trivial central idempotent of a ring $R$, and define

$$
A=\{x-e x \mid x \varepsilon R\}, \quad B=\{e y \mid y \varepsilon R\} .
$$

Then obviously (i) $A+B=R$. Next, since $e$ is central, (ii) each of $A, B$ is a two-sided ideal of $R$, and, since $e$ is non-trivial, (iii) neither $A$ nor $B$ is the zero ideal. Finally, if $z \varepsilon A \cap B$, say $z=x-e x=e y$, then

$$
z=e y=e^{2} y=e z=e(x-e x)=\left(e-e^{2}\right) x=0,
$$

i.e. (iv) $A$ and $B$ have no non-zero element in common. Thus $R$ is directly reducible and the proof is complete.

\section{Results for $\pi$-regular rings.}

We recall McCoy's definition [14]: an element $x$ of a ring $R$ is called $\pi$-regular in $R$ if a positive integer $s=s(x)$ and an element $b=b(x)$ of $R$ exist satisfying $x^{s}=x^{s} b x^{s}$. The ring $R$ is itself called $\pi$-regular if every element of $R$ is $\pi$-regular in $R$. The class of $\pi$-regular rings includes all algebraic rings and algebras (see [6]), and also all rings with minimal condition on left (or right) ideals. We now introduce a rather weaker

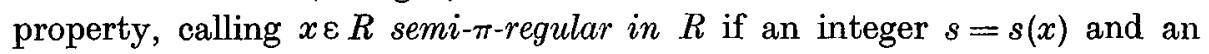
element $g=g(x)$ of $R$ exist satisfying either $x^{s}=x g x^{s}$ or $x^{s}=x^{s} g x$; and, as before, we call $R$ semi- $\pi$-regular if every element of $R$ is semi- $\pi$-regular in $R$. This definition is, clearly, again left-right symmetric; we note also that semi- $\pi$-regular rings share with $\pi$-regular rings the property of having nil Jacobson radical (this being very easily seen by the same argument as for the $\pi$-regular case).

We recall next that an element $x$ of $R$ is called strongly regular in $R$ if an element $a=a(x)$ of $R$ exists satisfying $x=x^{2} a, R$ being itself called 
strongly regular if every element of $R$ is strongly regular in $R$. The asymmetry of this definition is of course only apparent, by virtue of

LEMMa 1. If, to each element $x$ of a given ring $R$, there corresponds an element $a=a(x)$ of $R$ such that $x=x^{2} a$ or $x=a x^{2}$, then $R$ is strongly regular.

Proof. In fact $x=x^{2} a=a x^{2}$ for each $x$. For, if we are given (say) $x=a x^{2}$, then $\left(x-x^{2} a\right) x^{2}=x^{2}\left(x-a x^{2}\right)=0$, so that, since each monomial in the expansion of $\left(x-x^{2} a\right)^{2}$ begins with a factor $x^{2}$, we have $\left(x-x^{2} a\right)^{3}=0$. But clearly $R$ cannot contain any non-zero nilpotent elements, and so we deduce that $x=x^{2} a$, as required.

Our next theorem does not directly involve any questions of centrality, but we include it for the sake of its connexion with our later results.

Theorem 4. Let $R$ be any given semi-n-regular ring. Then the following statements are equivalent:

(i) $R$ is strongly regular;

(ii) $R$ is a subdirect sum of division rings;

(iii) $R$ contains no non-zero nilpotent elements.

Proof. That (i) implies (ii) is well known, and trivial in view of Jacobson's structure theorem for semi-simple rings (see also [7] for an elementary proof); and obviously (ii) implies (iii). Indeed, these two implications hold independently of the semi- $\pi$-regularity hypothesis. Thus we need only show that (iii), together with semi- $\pi$-regularity, implies (i); i.e., by Lemma 1 and the symmetry of semi- $\pi$-regularity, we need only show that, given $x, g \varepsilon R$ and a positive integer $s$ such that $x^{s}=x^{s} g x$, then we can, with the aid of (iii), construct $a \varepsilon R$ such that $x=x^{2} a$.

In fact we can take $a=g$. For if $s \geqslant 2$, then (with a suitable conventional interpretation when $s=2$ ) we have

$$
\left(x^{s-1}-x^{s-1} g x\right)^{2}=\left(x^{s-2}-x^{s-1} g\right)\left(x^{s}-x^{s} g x\right)=0,
$$

whence, by (iii), $x^{s-1}=x^{s-1} g x$, and consequently, by downward induction on $s$, we find that $x=x g x$. Hence $\left(x-x^{2} g\right) x=x(x-x g x)=0$, so that $\left(x-x^{2} g\right)^{2}=0$, and (iii) gives $x=x^{2} g$, as required.

The equivalence of (i) with (iii) in any algebraic algebra or ring with minimal condition on left ideals has been shown previously by Arens and Kaplansky ([1], Theorem 3.3); however, since each of these 
hypotheses implies $\pi$-regularity (and hence semi- $\pi$-regularity), their result is included in Theorem 4 above. It should be mentioned also that, at least in its specialisation to $\pi$-regular rings, Theorem 4 is implicit in [6] (cf. also [10], [11], Theorem 4 and [13], Theorem $5.5(\mathrm{e})$ ).

Theorem 5. Let $R$ be any given $\pi$-regular ring. Then the following statements are equivalent:

(iv) to each $x \in R$ there corresponds $a(x) \varepsilon R$ such that $x-x^{2} a(x)$ is central;

(v) to each pair $x, y \in R$ there corresponds $a(x, y) \in R$ such that $\left[x-x^{2} a(x, y), y\right]=0$;

(vi) $R$ is a $C N$-ring.

Further, each of these three statements is implied by any one of (i), (ii), (iii) above, and, by Theorem 1, any one of the six statements implies that $R$ is a CI-ring.

Proof. Since (iii) obviously implies (vi) and $\pi$-regularity implies semi- $\pi$-regularity, we need only prove the equivalence of (iv), (v) and (vi). Of these, (iv) implies (v) trivially, and (v) implies (vi) by Theorem 2, so it will be enough to show that (vi) implies (iv).

Now, given (vi) and any $x \in R$, say with $x^{s}=x^{s} b x^{s}$, then $\left(x-x^{s+1} b\right) x^{s}=x\left(x^{s}-x^{s} b x^{s}\right)=0$, while for a suitable $d \varepsilon R$, we may write $\left(x-x^{s+1} b\right)^{s}=x^{s}+x^{s} d$. Hence $\left(x-x^{s+1} b\right)^{s+1}=0$, and so (vi) makes $x-x^{s+1} b$ central, and (iv) follows with $a(x)=x^{s-1} b$.

COROLLARY. The property of being a $\pi$-regular $C N$-ring is preserved under homomorphism.

It is not hard to see that, even under the weaker hypothesis of semi$\pi$-regularity, say with $x^{s}=x^{s} g x$ for a given $x \varepsilon R$, (vi) implies that $x-x g x$ is central (so that $x^{s}=x^{m} g x^{n}$ for every pair of positive integers $m, n$ with $m+n=s+1)$, but it does not seem possible to deduce (iv) or ( $v$ ) from this.

The three conditions of Theorem 4 are of course (consider for example the special case of nil rings) not implied by those of Theorem 5 for general $\pi$-regular rings, and neither need any of these six conditions hold in a given $\pi$-regular $C I$-ring. To conclude this section, we note also the following 
result (which is appropriately mentioned here, since every algebraic algebra is certainly $\pi$-regular) :

THEOREm 6. Let $F$ be any given algebraically closed field of prime characteristic $p$, and let $R$ be any given algebraic $C I$-algebra over $F$. Then, for each $x \varepsilon R$, we can find a corresponding positive integer $r=r(x)$ such that $x^{p^{r}}$ is central.

Proof. Since $R$ is algebraic, every element $x$ of $R$ generates a finitedimensional subalgebra, and, since $F$ is algebraically closed, consequently (by the theory of the classical canonical form of a matrix) we can express $x$ as the sum of a nilpotent element of $R$ and a linear combination (over $F$ ) of idempotents, say $x=f+\Sigma \alpha_{i} e_{i}$. Then, since $F$ has characteristic $p$ and the $e_{i}$ are central, we have

$$
x^{p^{r}}=f^{p^{r}}+\Sigma \alpha_{i}^{p^{r}} e^{p^{r}}=f^{p^{r}}+\Sigma \alpha_{i}^{p^{r}} e_{i} \quad(r=1,2, \ldots),
$$

and the theorem follows on taking $r$ so large that $f^{p^{r}}=0$.

We remark that, by a result of Kaplansky [9], the conclusion of Theorem 6 in turn implies that the two-sided ideal $R_{1}$ generated in $R$ by all commutators $[u, v]$ must be nil ; if $R$ is even a $C N$-algebra, then of course (cf. [6], Theorem 5.7 or [15], Lemma 3) $R$ must actually be commutative (i.e. $R_{1}=0$ ).

\section{Regular rings.}

To round out the picture, we remind the reader that, independently of any side condition, strong regularity of a ring always implies regularity (i.e. the special case of $\pi$-regularity in which each $s(x)=1$ ): for, if $x=x^{2} a$, then $x(x-x a x)=\left(x-x^{2} a\right) x=0$, so that $(x-x a x)^{2}=0$, and consequently, if the element $x-x a x$ is also strongly regular, then $x=x a x$. In particular (as appeared in the course of the proof) semi- $\pi$-regular rings satisfying the conditions of Theorem 4 are in fact regular. We now prove

Theorem 7. For any regular ring $R$, each of the six conditions (i), ..., (vi) in Theorems 4 and 5 is equivalent to the statement that $R$ is a CI-ring.

Proof. Since every regular ring is $\pi$-regular, and since each (except the last) of the properties (i), ..., (vi) has already been shown to imply its successor in any $\pi$-regular ring, it follows from Theorem 1 that we need only show that the $C I$-property, together with regularity, implies (i). Now, if $x=x b x$ is any given regular element of $R$, then $x b$ is idempotent and hence central by the $C I$-property, whence $x=x^{2} b$, i.e. $x$ is strongly regular and the theorem follows. 
CoRollary. If $R$ is a regular $C I$-ring, then so is every homomorphic image of $R$.

Forsythe and McCoy [7] obtained the conclusion of Theorem 4 under the stronger hypothesis that $R$ is regular. They first proved the equivalence of (i) and (iii) directly, and then that of (ii) and (iii); the first equivalence is relatively trivial, while their method of proving the second (which used the equivalence of (i) and (iii), besides also Theorem 3 above and Birkhoff's structure theorem) can readily be extended to $\pi$-regular rings. See also [12].

[Added 15th March, 1957. It has come to the writer's attention that the equivalence, in regular rings, of (i), (ii), (iii) with the $C I$-property (and also with certain other properties not discussed here) was noted earlier by M. C. Waddell ("Properties of regular rings", Duke Math. J., 19 (1952), 623-627); however, he obtains no corresponding result for $\pi$-regular rings. We take this opportunity of remarking also that a result substantially better than Theorem 2 above has recently been found by Y. Utumi: see Lemma 1 of his paper " On $\xi$-rings", Proceedings of the Japan Academy, $33(1957), 63-66$.

\section{Annihilators in rings with minimal condition.}

THEOREM 8. Let $R$ be a ring with minimal condition on left ideals, and $A$ any non-zero left $C I$-ideal. Then $A$ either contains a non-zero twosided A-annihilator or has a two-sided identity.

Proof. As is well known (see for example [2], Theorem 2.6B) the hypotheses imply the existence of an element $e$ of $A$, and a nilpotent left ideal $M$ of $R$, such that

$$
A=A e+M, \quad e^{2}=e, \quad M e=0
$$

(where $e=0$ if and only if $A$ is nilpotent).

If $M=0$, then $A=A e$, and so $e$ is non-zero and (being central in $A$ ) acts as a two-sided identity in $A$.

If $M \neq 0$, then we can choose a positive integer $m$ such that $M^{m} \neq 0$, $M^{m+1}=0$. Then, for any non-zero $y \in M^{m}$, we have (since $e$ is central in $A$ and $M \leqslant A$ )

$$
\begin{array}{ll}
A y \leqslant(A e+M) M^{m}=A e M^{m}=A M^{m} e=0 \\
\text { and } \quad y A \leqslant M^{m}(A e+M)=M^{m} A e=M^{m} e A=0 ;
\end{array}
$$

thus $y$ is a two-sided $A$-annihilator. 
TheOREM 9. Let $R$ be a ring with minimal condition on left ideals, and $A$ any proper left $C I$-ideal. Then (i) $R$ contains a non-zero left $A$-annihilator. If, further, $R$ has a right identity 1 , then (ii) $R$ contains a non-zero right $A$-annihilator $z$.

Proof. (i) If $A=0$ then any non-zero element of $R$ serves as an $A$-annihilator. Otherwise the conditions of Theorem 8 are satisfied and so we may suppose that $A$ contains a two-sided identity $e$. Since $e$ is in the proper left ideal $A$, we have $R e \leqslant R A \leqslant A<R$, and so we can find an element $b$ of $R$ not representable in the form $b=c e$ with $c \varepsilon R$. Then $b-b e \neq 0$, while also, since $e$ is an identity for $A$,

$$
(b-b e) A=(b-b e) e A=0 ;
$$

thus $b-b e$ satisfies our requirements.

(ii) If $A=0$, take $z=1$. For $A \neq 0$, we again use Theorem 8. If the first alternative in Theorem 8 holds, take this annihilator as $z$; otherwise $A$ has an identity $e$ and we may take $z=1-e$ (this being non-zero since $R e<R=R 1$ ).

In Theorem 8, if $R$ is itself a $C I$-ring, then $e$ is in the centre of $R$, and so also is any right identity 1 of $R$, so that the annihilators constructed in Theorem 9 (i) and (ii) are then both two-sided; more generally, the $A$-annihilator $z$ of (ii) will in any case be two-sided if 1 acts as a two-sided identity on $A$. However, we cannot, even for commutative $A$, always assert the existence of a right $A$-annihilator in $R$; to see this, consider the (associative) ring $R$ generated by elements $x, y$ subject to the rules $x^{2}=x, x y=y, y x=y^{2}=0$, and take for $A$ the subring (clearly in fact a left ideal) generated by $x$.

There seem to be no easy generalisations of these results for arbitrary left ideals $A$. For, even if we require $A$ to be a proper two-sided ideal of $R$, then $A$ can nevertheless be without any one-sided identity (even in $R$ ) and at the same time be without any one-sided annihilator (even in $R$ ). As an example of this, consider the algebra $R$ of all $4 \times 4$ triangular matrices, over a field $F$, with a zero in the second diagonal place, and take for $A$ the ideal consisting of all members of $R$ having a zero also in the third diagonal place.

Also, of course, the minimal condition cannot be dropped, even for commutative $R$; for example, the conclusions of Theorems 8 and 9 both fail if $R$ is the complete direct sum of an enumerable infinity of fields and $A$ is the restricted direct sum. However, it does seem worth mentioning here a related result which holds without any assumptions on $R$ (for an analogous result about two-sided ideals, $c f .[10], 70)$ : 
Theorem 10. Given any ring $R$ and any left ideal $A$ of $R$, then either (i) $R$ contains a non-zero left $A$-annihilator, or (ii) the centraliser $C(A)$ of $A$ in $R$ coincides with the centre $Z$ of $R$.

Proof. Of course $C(A) \geqslant Z$, so that we have only to show that the negation of (i) implies the opposite inclusion. Take any $x \in C(A)$ and any $a \varepsilon A$. Then, since $A$ is a left ideal of $R, y a \varepsilon A$ for all $y \varepsilon R$, so that

$$
[x, y] a=x(y a)-y(x a)=(y a) x-y(x a)=y[a, x]=0,
$$

i.e. $[x, y] A=0$ for all $y \varepsilon R$; thus, if (i) is false, then $[x, y]=0$ for all $y \varepsilon R$, i.e. $x \in Z$. Since $x$ was an arbitrary element of $C(A)$, this means that $C(A) \leqslant Z$, as required.

Trivial though the results of this section are, they do not seem to have been pointed out before. However, Baer [3] has noted the special case of Theorem 8 in which $R$ is commutative and $A=R$ (cf. also [4], Lemma 7). And the case of Theorem 9 in which $R$ is a commutative finite-dimensional algebra with an identity element has been obtained, in a quite different way, by H. Schneider (unpublished).

\section{REFERENCES.}

[1] R. Arens and I. Kaplansky, "Topological representation of algebras", Trans. American Math. Soc., 63 (1948), 457-481.

[2] E. Artin, C. J. Nesbitt and R. M. Thrall, Rings with Minimum Condition (University of Michigan, 1944).

[3] R. Baer, "Inverses and zero-divisors ", Bull. American Math. Soc, 48 (1942), 630-638.

[4] N. Divinsky, "Pseudo-regularity", Canadian J. Math., 7 (1955), 401-410.

[5] M. P. Drazin, "Engel rings and a result of Herstein and Kaplansky ", American $J$. Math., 77 (1955), 895-913.

[6] —-, "Algebraic and diagonable rings", Canadian J. Math., 8 (1956), 341-354.

[7] A. Forsythe and N. H. MeCoy, "On the commutativity of rings", Bull. American Math. Soc., 52 (1946), 523-526.

[8] I. N. Herstein, "The structure of a certain class of rings", American J. Math., 75 (1953), 864-871.

[9] I. Kaplensky, "A theorem on division rings", Canadian J. Math., 3 (1951), 290-292.

[10] - "Topological representation of algebras II ", Trans. American Math. Soc., 68 (1950), 62-75.

[11] _- "Semi-simple alternative rings", Portugaliae Mathematicae, 10 (1951), 37-50.

[12] L. Kovács, "A note on regular rings", Publ. Math. Debrecen, 4 (1956), 465-468.

[13] J. Levitzki, "On the structure of algebraic algebras and related rings", Trans. American Math Soc., 74 (1953), 384-409.

[14] N. H. McCoy, "Generalized regular rings", Bull. American Math. Soc., 45 (1939), $175-178$.

[15] J. E. McLaughlin and A. Rosenberg, "Zero-divisors and commutativity of rings", Proc. American Math. Soc., 4 (1953), 203-212.

Department of Mathematics, North Western UnIVERSITY, Evanston, ImLinois, U.S.A. 